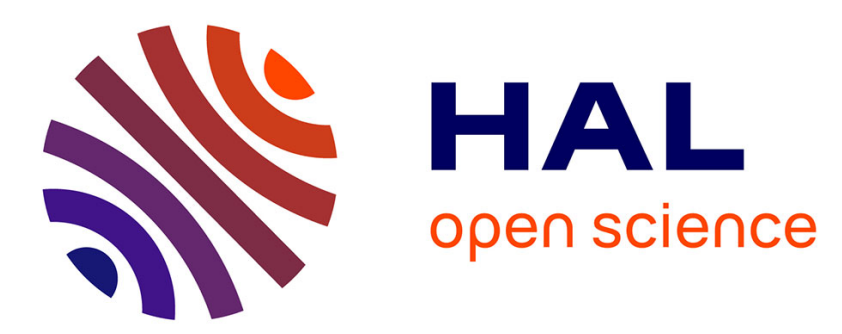

\title{
Les services publics d'eau face à la vulnérabilité sociale des populations: vers un nouveau modèle de management des services publics essentiels?
}

M. Tsanga Tabi

\section{- To cite this version:}

M. Tsanga Tabi. Les services publics d'eau face à la vulnérabilité sociale des populations: vers un nouveau modèle de management des services publics essentiels?. Flux - Cahiers scientifiques internationaux Réseaux et territoires, 2009, 76/77, p. 94 - p. 109. hal-00457607

\section{HAL Id: hal-00457607 \\ https://hal.science/hal-00457607}

Submitted on 17 Feb 2010

HAL is a multi-disciplinary open access archive for the deposit and dissemination of scientific research documents, whether they are published or not. The documents may come from teaching and research institutions in France or abroad, or from public or private research centers.
L'archive ouverte pluridisciplinaire HAL, est destinée au dépôt et à la diffusion de documents scientifiques de niveau recherche, publiés ou non, émanant des établissements d'enseignement et de recherche français ou étrangers, des laboratoires publics ou privés. 


\title{
Les services publics d'eau face à la vulnérabilité sociale des populations: vers un nouveau modèle de management des services publics essentiels?
}

\author{
Marie Tsanga Tabi
}

\begin{abstract}
"C'est le propre d'une société saine que de favoriser le développement de la solidarité entre ses membres" Émile Durkheim, De la division du travail social, 1893.
\end{abstract}

\section{INTRODUCTION}

'idée du développement durable est intéressante dans le -monde des services publics urbains dans la mesure où elle attire l'attention sur les inévitables tensions inhérentes aux dimensions économiques, environnementales et sociales de la gestion des organisations (Aggeri, 2001). Les difficultés des acteurs à mettre en œuvre les exigences d'une croissance économique autoentretenue tout en maintenant " l'accessibilité de tous à l'ensemble des biens et services, le renforcement des capacités de toute sorte et l'équité face à l'ensemble des ressources disponibles et transmissibles " en sont une illustration (Dubois et Mathieu, 2002). Dans le cas des services publics d'eau, la logique technico-économique à l'œuvre a généré des hausses du prix qui ont eu pour effet d'accroître les impayés et de réduire l'accessibilité financière au service d'une catégorie $d^{\prime}$ usagers subissant en outre les effets d'une précarisation croissante de ses conditions de vie. Ainsi, dans un contexte où paradoxalement l'on a atteint un niveau d'équipement exceptionnel en infrastructures de réseaux, les services publics d'eau à partir du milieu des années 1990 vont se trouver confrontés «malgré eux » à une population de plus en plus vulnérable sur le plan financier et social. Comment les acteurs de l'eau (1) vont-ils réagir? Quelles en seront les répercussions sur le plan organisationnel et managérial? Ces questions serviront de fil conducteur à notre réflexion, mais nous évoquerons en préalable les éléments de contexte à l'origine de cette situation. Nous terminerons notre propos par une discussion sur les questions de fond que soulève cette problématique nouvelle de la vulnérabilité des usagers d'eau.

\section{LA MONTÉE DU PHÉNOMÈNE DE VULNÉRABILITÉ SOCIALE DES POPULATIONS DANS LE SECTEUR DE L'EAU: ÉLÉMENTS D'ANALYSE ET D'APPRÉCIATION}

Avant de considérer les raisons et les symptômes de la montée de la vulnérabilité dans le secteur de l'eau, précisons le contenu de la notion de « vulnérabilité sociale » utilisée dans notre article.

Les éléments analytiques: la double dimension de la vulnérabilité des populations dans le contexte des services publics d'eau.

Pendant longtemps, la définition de la vulnérabilité des populations a renvoyé au concept de pauvreté monétaire. Cependant, les catégories analytiques de la vulnérabilité se sont enrichies en France, notamment avec les travaux de Lenoir (1974) et Castel (1995) qui ont développé la notion d'exclusion sociale. Cette notion au caractère multidimensionnel rend compte certes de la faiblesse de revenu, mais aussi de la déficience dans plusieurs domaines de sociabilité, l'emploi, l'éducation, le logement, les soins de santé, l'accès aux services publics essentiels dont l'eau. Aussi, la notion de vulnérabilité pour être bien comprise, rend-t-elle compte de différentes formes de fragilité et d'instabilité chroniques qui peuvent conduire à la précarité et pire encore à l'exclusion de la société (Paugam, 1991).

Cette approche sociologique de la vulnérabilité a besoin d'être complétée par les caractéristiques propres au secteur de l'eau pour mieux l'appréhender dans le domaine de l'accès à l'eau. En premier lieu, la rareté de la ressource ou le déficit en infrastructures n'est pas sans conséquences sur le niveau de vul- 
nérabilité des populations (2). Par ailleurs, l'évolution du prix de l'eau en France (et dans bien d'autres pays d'Europe) a augmenté plus vite que l'inflation générale des prix, tandis que les inégalités liées à la disparité des prix se sont accentuées pour les populations défavorisées. Cette deuxième dimension de la vulnérabilité intrinsèque à l'eau a été étudiée dans différents travaux et traduite par un critère relativement simple, le critère d'accessibilité financière à l'eau (3) (Fitch and Price, 2002; Sawkins and Dickie, 2005) qui mesure le poids des dépenses en eau dans le budget des ménages. Ainsi, le seuil de 3\% (4) est-il considéré comme un indice de vulnérabilité en matière d'accès à l'eau. Reynaud (2007), dans une étude où il mesure les problèmes d'accessibilité à l'eau en France, s'appuie sur ce seuil et estime à 1160000 le nombre de ménages concernés en France.

\section{Les symptômes de la vulnérabilité sociale des usagers d'eau: recrudescence des impayés d'eau et institutionnalisation de leur prise en charge}

Deux éléments vont rendre visible la question de la vulnérabilité des usagers dans le domaine de l'accès à l'eau:

\section{La recrudescence des impayés d'eau}

Le constat d'une pauvreté qui ressurgit dans les pays riches et ce, sous des formes nouvelles (émergence des sans domiciles fixes (SDF), pauvreté des jeunes, des mères isolées, familles monoparentales, permanence et croissance de l'emploi précaire...), est établi malgré les systèmes de protection sociale. Si en France le taux de pauvreté a reculé entre 1996 et 2004 (5), en revanche les disparités de revenus se sont creusées et les formes de précarité accentuées. La population des SDF (6) en particulier, soit 100000 personnes, pose un véritable problème en matière de non-accès à l'eau.

D'un autre côté, la courbe du prix de l'eau en France n'a cessé d'évoluer à la hausse (voir statistiques de l'INSEE). Entre 1990 et 1994, l'évolution moyenne est supérieure à 10\%, puis passe à 7\% entre 1995 et 1997. Entre 1998 et 2001, l'indice moyen des prix de l'eau a augmenté de 3,7\% et s'aligne sur l'évolution de l'indice général des prix à la consommation des ménages (hors tabac). L'augmentation est plus forte sur la période 2001 à 2005, l'indice des prix de l'eau a augmenté de 9,6\%, celui de l'assainissement de $12 \%$ alors que celui du coût de la vie reste inférieur $(8,2 \%)$.

De l'effet combiné de ces facteurs ont résulté des impayés d'eau dont l'ampleur inédite et soudaine va susciter des réac- tions fortes en provenance notamment du monde caritatif. Ainsi en 1999, sur les 20 régions où intervient le Secours Catholique, on comptait 15\% de cas d'impayés d'eau, soit 75989 personnes alors que 3 années avant, il n'en avait aidé que 45000. En 2003, I'INSEE dénombrait 120000 coupures d'eau, dont 2000 ayant duré plus de 24 heures et 17000 liées à une incapacité avérée de payer.

Cette montée en puissance des impayés et des coupures d'eau associées (environ 20000/an (7)) vont rendre visible la vulnérabilité des populations dans le secteur de l'eau. II s'avérera en effet un problème d'accessibilité au service d'eau traduit par le fait que les dépenses en eau dans le budget des ménages pauvres sont trois à quatre fois plus élevées que celles d'un ménage moyen français qui $n^{\prime} y$ consacre environ qu'1\% (Smets, 2007).

Un facteur d'une autre nature va renforcer la visibilité de la vulnérabilité des populations dans les services d'eau urbains: la loi relative à la solidarité et au renouvellement urbain du 13 décembre 2000 (8). L'individualisation progressive des compteurs d'eau qui en résulte va révéler des " poches géographiques » de précarité plus ou moins importante, en contexte $d^{\prime}$ 'habitat social notamment. Une étude sociologique menée à Nantes-Habitat met ainsi en exergue un nombre non négligeable de ménages aux revenus précaires: 23\% de référents en situation précaire et $18 \%$ de personnes inactives ou chômeurs recevant des allocations (Laumonier et alii, 2006).

\section{L'institutionnalisation de la prise en charge du problème des impayés}

À partir de 1992, l'inscription du problème des impayés d'eau dans l'agenda du politique contribue à la reconnaissance officielle du problème de la vulnérabilité des populations dans le secteur de l'eau. Une politique publique de maintien de la fourniture d'eau au profit des publics vulnérables connue initialement sous le nom de « dispositif solidarité eau » (9) est mise en place à partir de 1996. En 2004, ce dispositif est intégré au dispositif Fonds de Solidarité pour le Logement (FSL) (10), la politique sociale départementale en matière de maintien aux services publics essentiels (logement, eau, énergie et téléphone). II convient de noter également que la dernière loi sur l'eau et les milieux aquatiques (LEMA 2006) prévoit une série de dispositions juridiques nouvelles relatives à cette question. Nous y reviendrons quelques paragraphes plus loin. 
Face à ce phénomène de vulnérabilité des populations, on a vu les acteurs de l'eau s'impliquer à des degrés divers dans la mise en œuvre de solutions.

\section{LES SERVICES PUBLICS D'EAU FACE À LA VULNÉRABILITÉ SOCIALE DES POPULATIONS: LA CITADELLE TECHNIQUE CONFRONTÉE AU « NON- MARCHAND»}

Pour prendre la mesure des transformations en jeu, il importe de situer dans quels contexte et vision de l'action interviennent ces nouvelles préoccupations sociales.

\section{L'organisation des services publics d'eau: l'image de la " citadelle technique "}

S'il est un élément qui caractérise fondamentalement la catégorie des services publics en réseaux à laquelle appartiennent les services publics d'eau, c'est leur mode de fonctionnement selon le modèle de la « citadelle technique » (11) (Tsanga Tabi, 2003). La particularité de ce modèle d'organisation tient au déterminisme managérial des services d'eau qui fait intervenir substantiellement une logique technicienne à composante fortement réglementée ancrée sur une logique de territoire.

L'autre élément prégnant du modèle d'organisation des services publics d'eau tient à sa nature marchande qui régit les lois de l'accès au service selon le principe érigé en « paradigme de I'accès marchand » (12). Ce critère de l'accès marchand au service qui s'inscrit par ailleurs dans un univers de monopole local, légitime le point de vue technique à l'œuvre sur le plan fonctionnel et structurel. Les routines budgétaires et économiques s'auto-entretiennent selon une logique qui tire sa force d'un usager captif, doublée du poids de la contrainte réglementaire.

L'une des conséquences qui résulte de cet état de fait, c'est l'externalisation a priori des préoccupations du service, des questions qui ne relèvent pas du technique et en particulier celles qui relèvent du « social ». Aussi, le problème de la vulnérabilité sociale des usagers se pose-t-il en termes de défi aux services, en raison de "sa nature non technique et non marchande ».

C'est dans cet univers particulier et les représentations de I'action qui y sont associées que nous décrirons à présent les réactions des acteurs de l'eau et les effets induits sur l'organisa- tion du service. Nous distinguerons les réactions de l'acteur politique de celles de l'opérateur.

\section{Les acteurs politiques locaux face à la vulnérabilité sociale des usagers d'eau}

Poser le problème de la prise en charge de la vulnérabilité sociale des usagers d'eau du point de vue de l'acteur politique (l'élu local) n'est pas neutre car il interroge le registre sociopolitique de l'action des services et en cela pose la question de la responsabilité des impacts.

Sur un plan formel, l'élu est l'autorité publique légitime, l'acteur stratège qui conçoit le contenu et le niveau des missions du service public (rapport Denoix de Saint-Marc, 1996) en concertation avec les autres acteurs du système. II s'inscrit aussi dans le rôle de représentant du citoyen et de garant de l'intérêt général des usagers du service. À ce titre, l'autorité organisatrice est autant responsable de la satisfaction de l'usager solvable que de celle de l'usager en difficulté économique et sociale sur son territoire de desserte.

Sur un plan factuel, les réactions de l'acteur politique à l'exclusion des services publics essentiels sont diverses et relèvent en premier lieu de son degré d'implication dans le système d'acteurs. Le degré de formalisation de la politique publique locale de l'action sociale d'une part, et dans le domaine de l'eau d'autre part, influence par ailleurs le degré d'implication de l'élu. Quoi qu'il puisse en être cependant, on a vu les acteurs politiques locaux réagir dans deux directions.

\section{Les mesures d'interdiction de coupure d'eau pour cause d'impayés}

L'une des premières réponses de l'acteur politique à la vulnérabilité sociale des usagers d'eau a consisté à prendre des mesures d'interdiction de coupure d'eau. Quoiqu'on ne dispose que de peu de statistiques sur le sujet, la vague récente des arrêtés municipaux anti-coupures d'eau pris par les maires fait écho au problème posé par la vulnérabilité des usagers. Ainsi la ville de Vénissieux (mars 2006) et de Saint-Denis (novembre 2006), la commune de Champigny-sur-Marne (avril 2005), celles de Laudin-l'Ardoise et Sauveterre dans le Gard, Molezon et Sainte-Croix-Vallée-française en Lozère ont pris des arrêtés dans ce sens en 2007. Plus récemment, la Ville de Paris (mars 2008) annonçait qu'elle avait pris une mesure identique en faveur de ses usagers domestiques. 
Si une telle initiative semble relever a priori d'une prérogative reconnue aux élus locaux, il faut noter l'ambiguïté qui entoure les décisions prises dans ce domaine. En effet, plusieurs préfets ont déféré les arrêtés municipaux aux Tribunaux administratifs qui dans certains cas ont eu à les annuler au motif que ces décisions outrepassaient la compétence communale. Cependant, la controverse qui s'est instaurée autour de cette question sensible, et en particulier les cas d'entérinement (13) en hausse par les Tribunaux administratifs des arrêtés municipaux en question, tend à légitimer les initiatives de l'acteur politique (14).

Au-delà cependant, l'impopularité des coupures d'eau sur le plan politique joue en général en faveur des usagers d'eau. Beaucoup de collectivités locales, notamment celles qui sont en régie, ont tendance à ne pas couper l'eau des familles pauvres sans que cette indulgence ne relève d'un arrêté municipal formel.

De plus, les dispositions récentes de la LEMA votée en décembre 2006, de même celles qui sont à l'œuvre dans le cadre des missions des fonds de solidarité pour le logement (FSL) renforcent l'interdiction de coupure d'eau. Ainsi, l'article premier de la LEMA énonce " l'interdiction des coupures d'eau aux abonnés ayant bénéficié d'une aide au paiement des factures de la part du FSL ». Le décret d'application (15) (mars 2005) de la loi du 13 août 2004 relative au transfert de la prise en charge des impayés d'eau au FSL prévoyait déjà dans ses dispositions l'interdiction des coupures d'eau par les distributeurs du 1er novembre de chaque année au 15 mars de l'année suivante pour les familles qui bénéficient ou ont bénéficié, dans les 12 derniers mois, d'une décision d'attribution d'une aide du FSL.

Pour autant, les coupures d'eau pour cause d'impayés demeurent pour les cas de vulnérabilité non appréhendés au travers des critères d'attribution des aides (16), également lorsqu'il y a rejet de la demande d'aide ou en cas de non-recours au dispositif.

Les mesures de prise en charge des impayés d'eau dans le cadre des politiques d'action sociale locale

L'intervention des centres communaux d'action sociale (les CCAS), bras armé de la politique locale d'action sociale des maires, dans la prise en charge des impayés d'eau est antérieure à la parution des lois et décrets relatifs à l'exclusion des populations pauvres des services publics essentiels.

À la différence cependant des dispositifs départementaux, les réponses locales pour épurer les dettes d'eau ont toujours été facultatives dans la mesure où elles ne sont pas fléchées et qu'elles relèvent du pouvoir discrétionnaire de l'assemblée politique locale. S'il y a peu de retour quantifié de l'action des CCAS dans ce domaine, une étude menée en 2004 (17) sur l'action sociale des communes de 5000 à 200000 habitants indiquait que $64 \%$ des communes de cette catégorie étaient concernées par des apurements d'impayés d'eau.

L'existence du FSL au niveau départemental a cependant introduit l'idée d'un partenariat multi-acteurs dans la prise en charge de ce problème.

\section{Les opérateurs publics et privés des réseaux d'eau face à la vulnérabilité des populations}

La réaction des distributeurs d'eau face à la vulnérabilité des populations est à situer entre deux mouvements: I'un qui a consisté à intégrer le dispositif départemental de solidarité suite aux négociations menées au niveau national entre les représentants des acteurs de l'eau et l'État; l'autre plus local, où on a vu les distributeurs d'eau mettre en œuvre des solutions de partenariat avec les CCAS locaux. Nous illustrerons notre propos à la lumière de l'expérience de deux opérateurs, le service public d'eau de la ville de Vannes en premier lieu, régie municipale plus que centenaire, qui, dès 1992 et bien avant que la question des impayés d'eau n'apparaisse sur la scène publique, a mis en place un dispositif local d'identification et de prise en charge des impayés d'eau de ses usagers vulnérables (18). La Lyonnaise des Eaux sur le département du Loiret, dont l'expérience fut également pionnière, offre un second exemple dans un contexte de gestion déléguée de l'eau. Quoique l'étude ait été menée en 2002 et 2003, sa richesse sur le plan managérial illustre bien I'implication d'opérateurs d'eau dans la prise en main de ce dispositif (19).

L'exemple de la régie municipale de la ville de
Vannes: identification des publics vulnérables et
maîtrise des procédures de recouvrement des factures

À Vannes, ville portuaire, où la montée en puissance du pôle universitaire de Vannes-Lorient amène sur la région un flux ininterrompu d'étudiants, c'est l'effet conjugué d'un ensemble de facteurs qui amènent la ville de Vannes à prendre en comp- 
te la vulnérabilité sociale des populations dans son système d'action.

Les éléments de contexte: singularité de l'usager d'eau vannetais et montée des demandes d'aides en eau.

La singularité de l'usager d'eau vannetais tient à son statut généralisé d'abonné (20) hérité de l'histoire qui veut que tout habitant résidant en habitat collectif (21) dispose d'un compteur propre au même titre qu'un usager en habitat individuel. Ainsi, on compte 30000 abonnés d'eau pour une population de près de 58000 habitants, dont environ 16000 à 17000 abonnés qui relèvent de l'habitat collectif, soit un peu plus de la moitié (22).

Ce statut de l'usager n'est pas sans conséquences sur un plan financier et organisationnel. L'habitat collectif renvoie prioritairement à l'habitat social et potentiellement à la catégorie d'abonnés la plus fragile sur un plan économique. L'une des contraintes qu'engendre cette singularité contextuelle tient au risque d'impayé des factures estimé à 10\% des ventes d'eau (23). La gestion de ce risque est rendue d'autant plus complexe que la séparation ordonnateur - comptable, caractéristique de la gestion budgétaire en régie, implique une prise en charge externe (Trésor Public) des procédures de recouvrement des factures et de relance des impayés et que la régie doit malgré tout maîtriser.

Un deuxième facteur va contribuer à formaliser la prise en charge des impayés d'eau: l'accroissement des demandes d'aides qui, dans le seul domaine de l'eau, vont faire augmenter les aides accordées par le CCAS de 52\% entre 1990 et 1996.

Le dispositif solidarité Eau local de la ville de Vannes: un partenariat entre l'opérateur public, la Trésorerie et le CCAS La mise en place du « dispositif solidarité eau » local à Vannes date de 1992 mais dès 1990, le CCAS prenait déjà en charge des dettes d'eau. Face à l'enjeu financier qu'induit le risque d'impayés, le service de l'eau internalise la relance des impayés en négociant un accord en 1992 avec la Trésorerie, responsable du recouvrement des factures et des procédures de relance des impayés. Le partenariat vise avant tout la recherche de solutions en vue de maîtriser le processus de facturation et de recouvrement des factures d'eau. En parallèle, le CCAS saisit cette opportunité pour susciter une réflexion avec le service de l'eau sur l'idée de solidarité.
Ainsi, à partir de la liste des créances non recouvrées transmises par la Trésorerie, la régie de l'eau déclenche un processus de relance (envoi d'un courrier personnalisé indiquant le montant de la créance non soldée et le délai pour la régler) dont les différentes séquences aboutissent à des opérations de fermeture du compteur d'eau des usagers à une date annoncée en cas de non-règlement de la facture. Environ 50 fermetures de compteurs journalières sont programmées par tournée géographique. Quoique ce procédé soit impopulaire pour les représentations des usagers d'eau, il fait office de « moyen d'action incontournable » dans le service. Cette procédure est validée par le politique et garantit la crédibilité du service auprès des usagers en situation d'impayés.

Parallèlement à ce processus " interventionniste ", une action d'identification des usagers en précarité économique est déclenchée: la liste des impayés est transmise au CCAS qui se livre à une analyse de la situation de chaque abonné, fait une étude de dossiers et produit au service de l'eau une liste des cas d'impayés ayant un caractère social par tournée. Les abonnés en question sont alors retirés de la liste des impayés à relancer et entrent dans le dispositif "Solidarité Eau » local. Ce dispositif associe d'autres acteurs sociaux: des associations caritatives (Secours Catholique...), la CAF (Caisse d'Allocations familiales), la D.I.S. (Direction départementale de l'insertion sociale) et le Conseil général.

Sur un plan organisationnel, la contribution du service de I'eau à ce partenariat consiste à transmettre les informations relatives aux impayés d'eau afin de permettre au CCAS d'identifier les usagers éligibles au dispositif de solidarité qui représentent en moyenne $2 \%$ des usagers d'eau vannetais. La prise en charge financière des factures d'eau impayées cependant n'est pas totale, car le dispositif d'aide est plafonné.

L'opération "Hugo - énergie »: mensualisation de la facture d'eau et campagne de sensibilisation aux économies d'eau

Au début de l'année 1998, la flambée soudaine des demandes d'aide en eau potable et les difficultés à les financer par le budget de l'action sociale mettent en scène de nouvelles questions à résoudre: celle d'une part de la maîtrise pour le CCAS des aides octroyées et celle de la maîtrise par ailleurs des consommations d'eau des usagers non solvables. L'idée de la mensualisation des factures est suggérée par le service de l'eau mais sa faisabilité légale (24) se heurte à des exigences de procédures 
comptables de la part de la Trésorerie. Le CCAS insistera alors pour faire passer le nombre de facturations de 2 à 3 en dépit des réticences du service de l'eau qui met en avant l'argument de coûts supplémentaires. Des discussions entre partenaires qui suivront, naîtra l'idée d'une démarche de responsabilisation des usagers d'eau en difficulté associée à un passage à 3 factures dans le but de rendre ceux-ci plus attentifs à leurs consommations d'eau.

Une campagne d'information pour économiser l'eau, l'électricité et moins dépenser est déployée pour compléter le dispositif d'aide accordée aux familles. Un appartement éducatif « Hugo - énergie » équipé de tous les appareils électriques, à gaz et ceux associés aux usages en eau, est ouvert aux demandeurs d'aide et au public. Le CCAS est le chef de file de cette action menée en partenariat avec la Direction de l'eau et EDF, le Conseil régional, l'agence de l'eau Bretagne et le Ministère de l'environnement. La ville de Vannes est alors ville pilote pour les économies d'eau.

Sur la période 1998 à 2003, le taux de fréquentation moyen atteint les 450 personnes par an et une seconde version de guide pratique des économies d'eau est éditée par le service de l'eau.

L'impact de la campagne d'économies d'eau sur les consommations d'eau des usagers en difficulté et aidés par le CCAS reste difficile à évaluer en raison de l'absence de données détaillées. Le dispositif Hugo - énergie est toujours actif.

\section{La Lyonnaise des Eaux dans le département du Loiret: un exemple d'engagement des acteurs privés de l'eau}

À l'image de la régie d'eau de Vannes, I'implication initiale des distributeurs d'eau privés pour prendre en charge les impayés d'eau est locale et prend la forme de participations à des fonds locaux communaux dans certaines banlieues « à problèmes »:

" On avait effectivement mis en place également à cette époque-là, des fonds locaux dans les communes qui étaient les plus marquées. On s'était impliqué en tant que Lyonnaise des Eaux à Calais, à Villeneuve Saint-Georges, à Dreux; là où on avait des problèmes relativement importants de paiement. Dans d'autres cas, cela ne s'était pas concrétisé par des fonds réels, mais il y avait des démarches qui étaient initiées avec les services d'aide sociale des villes [...] Ces fonds ont vécu 2 ou 3 ans, on n'a pas vraiment eu le temps de les voir évoluer parce qu'il y a eu la Charte solidarité qui est arrivée » (entretien avec le responsable clientèle de la Lyonnaise des Eaux au niveau national, 2002).

L'institutionnalisation des dispositifs solidarité-Eau en 1996 conduit les principaux distributeurs privés d'eau à afficher une position de partie prenante officielle des différents dispositifs départementaux avec des degrés de réalisation de cette intention très disparates d'un département à un autre. Sur le principe, cette prise de position résulte de la forte implication (25) de la fédération des distributeurs d'eau privés (la FP2E) aux négociations qui ont entouré l'inscription sur l'agenda du politique du problème des impayés d'eau en 1995 et qui aboutira à la signature en novembre 1996 de la première version des dispositifs départementaux de Solidarité Eau (Charte Solidarité Eau du 6 novembre 1996).

En 2006, la FP2E indiquait que 18700 dossiers (26) avaient été traités à l'échelle nationale dans le cadre des FSL départementaux dans leur version actuelle (27), soit l'équivalent de 1,7 million d'euros d'abandons de créances (28) (rapport 2006 sur les services publics d'eau et d'assainissement).

Peu d'études ont été menées pour pouvoir apprécier l'impact de cette implication sur l'organisation des services. Nous illustrerons notre propos en retraçant ici l'expérience pionnière de la Lyonnaise des eaux sur le département du Loiret.

Caractéristiques de la vulnérabilité des usagers d'eau sur le département du Loiret sur la période étudiée

En 2002, la Lyonnaise des Eaux gérait 35 contrats de délégation de service public (52 communes) sur le département du Loiret représentant 80639 abonnés. Sur la période étudiée (19982001), les demandes d'aide concernent 1102 personnes soit $0,47 \%$ des abonnés du service.

Le public demandeur est en règle générale allocataire de revenus sociaux (allocations adulte handicapé, allocations chômage, allocations familiales, RMI). II s'agit notamment de familles monoparentales, couples divorcés, jeunes couples en situation de chômage ou en situation d'emploi précaire avec une part de foyers monoparentaux féminins qui représentent près du quart des demandeurs.

Si l'année 1998 marque le démarrage du dispositif (41 dossiers traités), l'évolution des dossiers traités entre 1999 et 2000 est de $+92 \%$ : on passe de 292 demandes à 560. Près d'une 
cinquantaine de dossiers est traitée chaque mois.

92\% des impayés d'eau se situent dans une classe de factures semestrielles allant de 171,66 à 446,68 €; soit 0,12 à près de trois fois supérieur à la consommation moyenne nationale (29). Ces niveaux d'impayés traduisent par ailleurs des effets prix qui renvoient aux disparités du prix de l'eau dans la région du Loiret. Sur le district de Montargis, le prix du $\mathrm{m}^{3} \mathrm{~d}^{\prime}$ eau est de $3,20 €$, tandis qu'à Orléans, il est 2,29 €. Dans certaines communes notamment en régie, le prix de l'eau est en dessous des $1,52 €$.

\section{Les modalités de fonctionnement du dispositif} solidarité-Eau sur le département du Loiret

Le principe de fonctionnement du dispositif repose sur la logique du mode partenarial où l'action conjointe des acteurs permet de réunir deux types d'expertise: une expertise sociale détenue par les représentants de l'action sociale et une expertise technique renvoyant au monde de l'eau. Le partenariat désigne alors " un collectif d'acteurs devant résoudre un problème. Dans ce schéma d'action, aucun acteur n'a le monopole des solutions concernant les populations en précarité. Ainsi, les solutions sont recherchées en commun. Des procédures de concertation doivent alors être mises en place. Juridiquement, cette action collective est formalisée par la signature de conventions entre les parties » (30). Cette définition mobilise un certain nombre d'idéaux et de formes de représentations de l'action à mettre en œuvre, notamment celles de l'action consensuelle et de l'économie solidaire.

Ce partenariat est activé par la mise en place d'une commission départementale, assemblée paritaire qui réunit une fois par mois entre 7 et 15 personnes représentant le directeur des actions interministérielles de la préfecture ainsi que son bureau de la ville et de la solidarité, certains services déconcentrés de l'État, les décideurs locaux de l'eau et de l'action sociale, les associations caritatives (Secours Catholique notamment) et la $\mathrm{FP} 2 \mathrm{E}$. La règle de représentation établie en interne par la FP2E repose sur la part de marché qu'occupe un opérateur donné sur le territoire, en l'occurrence, la Lyonnaise des Eaux dans le cas étudié. La commission départementale est ainsi l'outil principal de l'action qui vise 2 objectifs:

- aider les familles qui présentent des impayés d'eau potable et notamment « examiner les dossiers de demande d'aide et décider du montant de l'aide » accordée une fois par an sur la base du plafond de ressource autorisé (31);
- mettre en place des actions d'information et de pédagogie.

S'agissant des règles de contribution financière, chaque acteur de l'eau, repérable sur la facture par une quote-part de recette lui revenant, abandonne la part de créance qui le concerne (part FP2E et part État). Cela étant, cette règle ne vaut que si l'acteur en question (hormis l'État (32)) est signataire de la convention départementale. La nouvelle facture défalquée des abandons de créance est établie par les services d'eau tandis que l'aide accordée par « les acteurs du social » est directement envoyée au distributeur d'eau.

Les résultats du partenariat mis en œuvre Sur la période étudiée, la saisine du dispositif a été effective, 1120 dossiers ont été instruits et 32 réunions de délibération se sont tenues au rythme d'une réunion par mois avec une mobilisation moyenne des partenaires qui s'est maintenue tout au long des 3,5 années. Cela étant, le principe d'aide met en jeu des contributions inégales des acteurs et un taux de prise en charge des impayés d'eau relativement faible. L'aide moyenne par demandeur est de 93,45 € en 2000 et de 97,37 € en 2001 et se situe dans une fourchette d'aide accordée qui varie entre un minimum de $33,23 €$ et un maximum qui se situe à 466,29 €. Ramené à la demande d'aide, le taux global de prise en charge des impayés d'eau par le dispositif se situe respectivement à 50,5\% en 2000 et 47\% en 2001 .

En valeur absolue, l'acteur social (CCAS) qui se substitue de fait à la collectivité locale est celui dont la contribution au financement de l'aide aux impayés d'eau est la plus importante: $40 \%$ en 2000 et $42 \%$ en 2001 . Certes, les acteurs de l'eau (État+FP2E) à eux deux interviennent à hauteur de $60 \%$ mais leur effort de contribution est nettement moins marqué que celui de l'acteur social.

En effet, si on compare le taux d'intervention de chaque acteur eu égard à la quote-part de facture d'eau qui l'engageait, le contraste entre l'effort respectif des acteurs est frappant (voir graphique).

De fait, c'est l'engagement financier de l'acteur social (le CCAS) qui est le plus significatif, aussi bien vis-à-vis du montant global de l'aide accordée par le dispositif qu'en termes d'effort consenti, ceci pour deux raisons:

- le CCAS intervient de fait lorsque l'aide accordée par les acteurs de l'eau est négligeable ou que le dossier a été reje- 
té pour motif de dépassement de plafond de ressource;

- le CCAS prend intégralement en charge certains soldes de facture une fois que le distributeur privé et l'État ont abandonné leur quote-part de créances.

Quant à la FP2E, la règle de contribution s'appuie sur le texte de la circulaire d'application du dispositif, qui laisse le choix aux opérateurs entre un abandon partiel de sa créance et un abandon total. L'abandon partiel lui permet d'étaler son droit de tirage dans le temps et de minimiser son implication financière par rapport à l'engagement pris au niveau national. De son côté, l'État qui est censé « participer en abandonnant sa part correspondant aux taxes et redevances » (circulaire), prend le parti de ne pas abandonner la TVA.

Les délais d'intervention du dispositif quant à eux sont bien trop longs: entre le moment où un usager fait sa demande d'aide et le moment où il reçoit sa facture défalquée de l'aide accordée, il se passe environ entre 4 à 6 mois. Enfin, la couverture territoriale par le dispositif départemental reste en grande partie inachevée, même si cette situation n'exclut pas l'existence de dispositifs d'aide locaux. Sur la période étudiée, la greffe de la solution institutionnelle de l'aide aux impayés d'eau sur le département du Loiret ne s'est faite que pour un tiers des abonnés d'eau potable.

L'une des questions qu'il est intéressant d'examiner à ce stade de notre analyse est celle des impacts au sein des services publics d'eau. Cette expérience a-t-elle modifié les représentations des acteurs et le management du service d'eau? C'est ce volet que nous abordons à présent.

\section{Les effets de la prise en charge de la vulnérabilité des populations sur les représentations des acteurs et le management de la citadelle technique}

L'irruption du social dans la citadelle technique produit des évolutions observables au moins à trois niveaux:

Du point de vue des représentations portées par les acteurs en premier lieu, I'implication des services d'eau dans le dispositif FSL, et notamment la forme partenariale qu'elle revêt, représente une excellente opportunité de sortir la citadelle technique de sa logique isolationniste en lui donnant l'occasion de confronter sa vision technico-marchande de l'accès à l'eau à une approche non marchande de cet accès à l'eau.

En effet, à la différence du dispositif local vannetais qui
Taux de contribution des acteurs de l'eau au financement des impayés d'eau par rapport à la quote-part des créances initiales

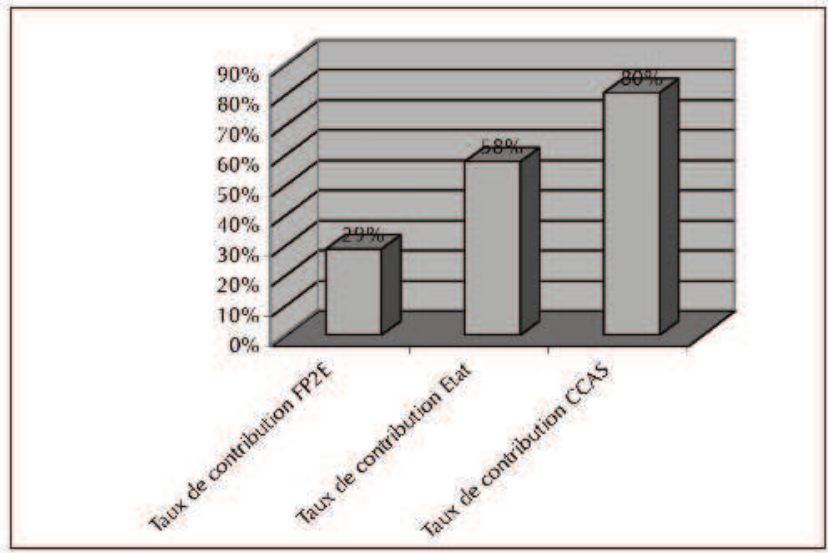

mobilise peu les acteurs de l'eau dans la prise de décision pour aider ou pas un usager, le modèle départemental initié dans le Loiret implique l'acteur de l'eau dans cette discussion. La mise en scène de la commission départementale apparaît alors comme un lieu favorable pour faire émerger les représentations individuelles qu'ont les acteurs de l'eau du problème de la vulnérabilité des populations et de ses modes de résolution. Parce que cette mise en scène bouscule les représentations dominantes du service public de l'eau, on serait tenté de croire que la confrontation des acteurs de l'eau à cet objet nouveau a initié un changement des perceptions des problèmes de vulnérabilité sociale et de leur prise en charge dans l'univers de la citadelle technique.

Cependant, l'heure n'est pas encore à une prise de conscience affirmée publique et collective du problème posé par la vulnérabilité sociale des populations, en particulier du côté des acteurs de l'eau. Un responsable clientèle d'un des principaux groupes privés nous faisait la remarque suivante:

"Tout le monde dit qu'il y a de plus en plus de fracture sociale. Pour ma part, $[\ldots]$ je fais très peu confiance aux statistiques parce qu'elles servent à des fins politiques. J'ai du mal à avoir une idée objective et sincère de la réalité. Ici, il est sûr qu'on accorde de plus en plus d'échelonnements des paiements » (entretien avec le responsable Véolia Eaux sur le département du Gers, 2002).

De fait, le quotidien de la citadelle technique, d'après les acteurs de l'eau, ne semble pas particulièrement mettre en avant les difficultés sociales. Il y a d'autres raisons plus pro- 
fondes cependant qu'il convient d'évoquer, qui tiennent aux représentations des acteurs et qui peuvent être source d'ambiguïté lors du diagnostic du problème. La campagne d'économies d'eau préconisée pour les usagers vannetais en difficulté financière est certes louable, mais elle n'en rejoint pas moins une forme répandue d'interprétations du problème dans le monde de l'eau. Pour le distributeur d'eau, la vulnérabilité sociale des usagers ne serait pas tant un problème de solvabilité qu'une question de maîtrise de ses consommations et de détection de fuites d'eau éventuelles.

«Plus on expliquera aux gens ce qu'est l'eau, qu'il faut l'utiliser et ne pas la gaspiller, qu'un budget d'eau ça revient de manière récurrente... Notre métier à nous, ce n'est pas de dire aux gens de consommer à tout prix. C'est de leur dire de vérifier ce qu'ils consomment de sorte qu'il n'y ait pas de problème. À partir du moment où les gens vérifient leur consommation d'eau, ils prennent conscience de ce que cela coûte. De plus, on met à leur disposition différents moyens de paiement, l'échelonnement, la mensualisation... et on les incite à prendre contact avec nous, à ne pas avoir peur de nous afin qu'on puisse répondre à leurs préoccupations, je pense que là on devrait identifier les gens qui ont de réelles difficultés » (entretien avec le responsable Lyonnaise des Eaux France des départements des Pyrénées Atlantiques, des Landes et Pays Basque, 2002).

C'est en référence à de tels diagnostics que l'on a vu se développer la mensualisation dans les services d'eau face à la vulnérabilité des populations. Toutefois, l'hypothèse que la confrontation des services à la vulnérabilité des populations modifie leur perception et leur analyse du problème de la vulnérabilité des populations n'est pas irréaliste ainsi que le laissait supposer le représentant de la Lyonnaise des Eaux dans l'expérience relatée.

"Ce qui me surprend le plus, c'est de voir la situation de certaines familles. Lorsqu'on rencontre encore de tels cas, c'est vrai qu'on se pose des questions » (D. Taillardat, responsable du contentieux du service clientèle de la Lyonnaise des Eaux sur le secteur d'Orléans (33), 2001).

Du point de vue des principes d'organisation des services publics d'eau en second lieu, l'expérience vannetaise marque le début d'un processus d'internalisation de la gestion des publics vulnérables, à souligner dans le cas des régies en particulier où le principe de séparation ordonnateur-comptable induit de fait une distance entre le service et ses usagers en difficulté. Si le fichier des abonnés des services d'eau regorge d'informations utiles concernant l'usager, la structure de ce fichier ne permet pas de faire de recoupements entre les informations relatives aux consommations et aux prix de l'eau par exemple et les caractéristiques socio-économiques des publics vulnérables. L'implication des services publics d'eau dans ces dispositifs sociaux a-t-elle amélioré leur connaissance de cette tranche d'usagers? Nous ne disposons pas de retours suffisants pour pouvoir en juger, mais il ne semble pas que ce soit le cas et quoi qu'il en soit, la question des effets de l'action mise en œuvre sur les publics cibles est laissée de côté. Ce qui est certain, c'est que la détention de ces informations permettrait de mieux argumenter les débats actuels sur la mise en place d'une tarification sociale dans le secteur de l'eau, tout comme elle permettrait d'objectiver davantage les diagnostics des acteurs de l'eau. À cet égard, l'on peut dire que les acteurs de la commission d'attribution des aides ont eu une approche très mécaniste de leur mission. La formule de l'abandon de créance qui encadre l'action apparaît comme un facteur limitant l'initiative des acteurs privés et là où la commission aurait pu innover, autour des conditions d'attribution de l'aide notamment, il n'y a pas de débat entre les acteurs pour affiner le comment de l'action. Par exemple, la variable " prix de l'eau » et les disparités géographiques associées, qui induisent une inégalité de traitement en amont du système d'aide, sont ignorées par les acteurs de I'eau en particulier.

Quoi qu'il en soit cependant, ces dispositifs légitiment la différenciation des usagers fondée sur le critère du revenu, dont le principe est en rupture avec la conception dominante dans le secteur des services publics à caractère industriel et commercial (34). En légalisant l'interdiction de coupure d'eau pour cause d'impayés et en prévoyant pour les publics vulnérables, un droit d'accès différencié au service destiné à compenser les inégalités (même si cela n'a lieu qu'une fois par an), le point de vue de l'égalité des usagers adopté par les acteurs est de fait plus utilitariste au sens de la théorie économique (Rawls, 1987) (35). On admet une définition de catégories à qui on doit assurer "que les inégalités sociales et économiques soient au bénéfice des plus désavantagés » (Affichard, 1995) et l'on peut penser que, pour cette raison aussi, "l'indifférence » des services publics d'eau vis-à-vis de ce segment d'usagers n'est pas tenable dans le temps. 
Du point de vue du territoire enfin, l'évolution la plus notable réside dans le changement d'échelle qu'induit la gestion de la vulnérabilité des populations eu égard à l'eau. L'échelle de territoire désignée par la politique de prise en charge des populations défavorisées se réfère formellement à la circonscription supra locale qu'est le département et se juxtapose à l'espace de référence initial de gestion du service public d'eau, qui lui est principalement communal ou intercommunal. Cela étant, cette approche du territoire supra local pour gérer la solidarité qui passe par l'engagement volontaire des acteurs de l'eau (l'adhésion à la convention) n'est pas partagée par ceux-ci.

En effet, si pour les acteurs privés de l'eau l'adhésion au dispositif départemental prolonge dans les faits une décision négociée, elle n'est pas synonyme d'adhésion à la vision du nouveau territoire d'action initié au niveau départemental. Lors de la mise en œuvre, chaque acteur en présence met en avant sa perception et sa définition du territoire d'action qui lui est propre. Muller (1996) et Palier (1998) ont souligné cette difficulté s'agissant des politiques sociales territorialisées: "les différents acteurs présents ont chacun leur référence spatiale ou territoriale, et ne s'en affranchissent pas dans une action qui ne parvient pas à être commune » (36).

II n'y a rien d'anodin dans le fait que la formule de l'abandon de créance soit celle qui ait été négociée et pratiquée par les distributeurs d'eau privés. Non seulement elle induit une discontinuité territoriale dans le cadre du nouvel espace d'action, mais de plus, elle ne garantit pas l'équité d'accès au dispositif entendue à l'échelle départementale. En effet, la particularité du principe de l'abandon de créance tient au fait qu'elle engage financièrement le distributeur d'eau uniquement à l'échelle du territoire de desserte en eau du service et non pas à l'échelle du territoire sous-jacent à la convention. Autrement dit, le distributeur d'eau ne contribue à la solidarité départementale (n'abandonne de créance) que dans la mesure où elle concerne un impayé d'eau relevant de « son » territoire de desserte. De plus, cette formule n'est pas la plus optimale pour la commission d'attribution des aides, car elle reproduit la complexité inhérente à la gestion de l'eau en s'attachant à identifier des quotes-parts de créance sur la base de factures d'eau peu lisibles par ailleurs.

À l'inverse, la formule de l'abondement direct au fonds, qui caractérise la contribution des autres acteurs partenaires (dont celle de certains distributeurs publics d'eau), s'affranchit de ce mode compartimenté de la prise en charge de l'aide. Elle repose sur un idéal de cofinancement de la solidarité à l'échelle du département et vise en particulier l'exigence de continuité territoriale fondée sur un principe de mutualisation du fonds.

Pour ce qui concerne les distributeurs d'eau publics telle la ville de Vannes, il est certain que l'idée d'une action re-territorialisée du service public d'eau pour gérer la solidarité se heurte au principe de libre administration des collectivités locales établi par la loi de décentralisation du 2 mars 1982. Le nouvel espace d'action pose d'emblée un conflit de compétences entre le local et le niveau supra local que traduit bien la formule « laissez-moi gérer mes pauvres » (37), et qui s'oppose de fait au principe de solidarité élargi à l'échelle départementale.

\section{DISCUSSION ET PERSPECTIVES: VOIT-ON ÉMERGER UN NOUVEAU MODÈLE DE MANAGEMENT DES SERVICES PUBLICS D'EAU CONTEMPORAINS?}

La portée du changement à l'œuvre dans le secteur de la gestion de l'eau du fait de la nouvelle donne sociale qui s'impose aux services relève d'une dynamique « lente ». Près de 20 ans après les premiers signes visibles de ce problème et 12 ans après la parution de la première charte Solidarité Eau, les solutions apportées à ce jour n'ont eu qu'une portée curative. Pour les acteurs de l'eau en particulier, ces solutions relèvent d'une logique facultative (38) et doivent viser prioritairement une inclusion de type marchande destinée à rétablir avant tout le lien marchand à la fourniture du service pour le segment de population étudié alors même que le fondement de ce lien pour les usagers en question se délite. Comment en effet continuer à concevoir l'accès à ce service essentiel sous I'angle de la seule logique marchande pour cette catégorie de population tombée dans une insécurité économique et sociale devenue structurelle? Pourtant, la relation non marchande qui s'instaure vis-à-vis de l'usager dans le cadre du partenariat avec le FSL initie une logique différente et entend participer — sur le principe tout au moins - à un processus d'inclusion sociale visant à « restaurer le droit des populations fragilisées à jouir d'un niveau de vie et de bien-être considéré comme normal pour la société dans laquelle elles vivent » (Commission Européenne, 2003). S'il est une question importante que soulève cette confrontation du service public d'eau à ce nouvel objet non marchand, c'est bien de savoir comment mieux prendre en compte ces populations 


\section{en termes d'accès et de maintien au service?}

À ce jour, en dehors des statistiques tenues sur les moyens mobilisés, on n'a quasiment pas de retour sur les effets de ces dispositifs en termes de préservation de l'accès au service. De fait, le modèle de la « citadelle technique » peine à muter et nombre de débats ne sont toujours pas tranchés. C'est notamment sur deux débats majeurs que nous voulons à présent engager notre discussion, celui concernant le statut de la solidarité dans la sphère des services publics d'eau et celui de la responsabilité que pose aujourd'hui aux acteurs l'enjeu contemporain de cohésion sociale.

\section{La solidarité " sociale ", objet nouveau du périmètre des services publics d'eau?}

L'un des débats de fond sous-jacent au problème d'exclusion des services publics d'eau des catégories étudiées renvoie à la définition de la solidarité et notamment à l'appréhension par les acteurs de ses formes nouvelles. Initialement, dans le monde très codifié de l'action des services publics d'eau, l'idée de la solidarité rural-urbain et du principe de cohésion territoriale sous-tendu n'a pas posé de problèmes majeurs d'un point de vue opérationnel, et pas davantage sur le plan financier. En effet, la concrétisation de l'idéal de solidarité en question voulait que les usagers ruraux bénéficient de l'accès à l'eau au même titre que les usagers urbains et cela, indépendamment de la différence de situation liée aux coûts de desserte. Cet idéal a trouvé sur le plan opérationnel des supports familiers et naturels dans le champ des services publics d'eau, à savoir le réseau et les subventions croisées résultant des péréquations tarifaires appliquées sur les territoires politiques de desserte.

D’un point de vue économique, la réalisation de cette idée a valu de déroger à la norme économique de tarification au coût marginal considérée alors comme la seule efficace en contexte de monopole (Boiteux, 1986). On a ainsi adjoint au prix de l'eau, une taxe additionnelle perçue sur chaque $\mathrm{m}^{3}$ d'eau facturé et qui a servi à financer le FNDAE (Fonds National D'Adduction en Eau potable) supprimé par la loi de Finances rectificatives 2004-1485 du 30 décembre 2004 (39).

La prise en compte ensuite des préoccupations environnementales dans la sphère d'appréhension des missions du service public d'eau rend compte d'un élargissement progressif des contours du service public d'eau: les taxes prélevées par les agences de l'eau sont assises sur le prix de l'eau et ont vocation à financer la préservation et la dépollution des ressources en eau. En 2007, elles représentaient 18\% des recettes issues des ventes d'eau à l'usager (BIPE/FP2E, 2008).

Notons par ailleurs que l'idée de solidarité votée par la loi Oudin le 27 janvier 2005, qui autorise les services publics d'eau à financer sur leurs budgets des actions de coopération décentralisée (40), participe à ce mouvement d'élargissement du périmètre du service public. Cette nouvelle forme de solidarité entre services d'eau des pays riches et services d'eau des pays pauvres rejoint la solidarité associée à la vulnérabilité des usagers démunis en France.

De fait, I'analyse des flux économiques liés à l'eau témoigne clairement d'un élargissement progressif du périmètre formel du service public d'eau: ceux-ci ne sont pas exclusivement dédiés à l'eau dans sa composante service, mais ils intègrent des dimensions plus larges que le strict service fourni à l'usager.

Dans le même état d'esprit, les débats actuels sur la tarification sociale de l'eau, ou d'un éventuel droit à l'eau pour garantir durablement le maintien de l'accès à l'eau des publics vulnérables, relèvent d'une problématique identique. Sur le plan de la doctrine française du service public cependant, et s'agissant notamment des missions dévolues aux services publics (qu'ils soient marchands ou non), il est clair que «le premier des biens publics est sans doute ce qu'on appelle « la cohésion " sociale, ou encore la capacité d'une société à « tenir » ensemble » (Strobel et Kaisergruber, 1996). Parce qu'ils sont insérés dans la société, les services publics d'eau ne peuvent pas ignorer ou sous-estimer la dimension sociale et l'exigence amplifiée de justice sociale et d'équité qui s'attachent à la fourniture contemporaine de ce service essentiel. S'il fut question au cours du XIXe siècle de faire face au défi de santé publique et de desserte en eau par le réseau, I'enjeu de la solidarité sociale est celui que doivent relever les acteurs de l'eau du XXIe siècle. Cela oblige les acteurs de l'eau à repenser la relation entre différences sociales et service public pour concevoir des prestations qui soient simultanément justes et efficaces d'un point de vue économique.

\section{À qui revient la responsabilité de la cohésion sociale dans la sphère des services publics d'eau?}

L'autre question de fond que la confrontation des services publics d'eau à la vulnérabilité sociale des populations met en 
exergue, est celle de la responsabilité de sa prise en charge dans le monde de l'eau. Certes, sur un plan doctrinal, il est établi que "les services publics sont un fondement de la cohésion de la société (41). [...] Fondamentalement, les services publics sont faits pour satisfaire leurs utilisateurs et pour assurer en même temps la cohésion sociale » (42) (Denoix de Saint Marc, 1996) (43). Pour autant, s'agissant de savoir qui en est responsable, les acteurs n'arrêtent pas de se renvoyer la balle.

\section{La responsabilité de la cohésion sociale dans le secteur de l'eau incombe à la puissance publique}

Bien qu'elle soit souvent posée en termes financiers ou en termes d'implication des acteurs, la question de la responsabilité de la prise en charge des questions sociales liées à l'eau relève avant tout de la vision du monde portée par chaque acteur et que Muller (2006) désigne par la notion de « référentiel » (44). Les distributeurs privés d'eau en sont bien conscients:

"Le débat collectif sur la solidarité dans le secteur de l'eau est une vraie question qui mériterait d'être renvoyée au politique. Dans ce système, chacun essaie de proposer la solution qui lui semble la plus efficace, la plus simple à gérer, mais sans qu'il y ait de réelle ambition derrière. On doit chacun à sa place se poser la question de sa mission. C'est vrai que pour une entreprise, sa mission, c'est de chercher à équilibrer sa gestion du service à la fois pour les clients mais aussi pour dégager un bénéfice, sinon elle n'existe pas. Elle est bien obligée quelque part de prendre en compte son environnement qui, lui, est défini par la loi, les règlements $[\ldots] \mathrm{Si}$ on nous fait une réglementation sur la tarification, ce sera une donnée d'entrée. Mais en tant qu'entreprise, ce n'est pas à nous de définir ce que doit être le social... » (entretien avec le responsable clientèle du groupe SAUR, 2001).

Cet extrait d'entretien recadre bien la question de la responsabilité qui sur le fond n'est pas tant une question financière. Le vrai problème est bien celui de la prise en compte des effets sociaux du fonctionnement des services publics d'eau. $C^{\prime}$ est justement ce que suggère l'analyse des politiques publiques ou encore l'approche en termes de développement durable. De ce point de vue, il est clair que la responsabilité des effets des politiques publiques locales incombe à l'acteur politique, investi de la puissance publique (Duran, 1996; Thoenig, 1981), et autorité organisatrice du service public d'eau même si, nous le voyons plus haut, la position du législateur et la jurisprudence à ce sujet ne sont pas claires.
Pour autant, les évolutions juridiques en cours (article 1er de la LEMA, 2006) (45) laissent entrevoir de nouvelles perspectives (46), dont celle de mettre en œuvre des formes de prise en charge « amont » plus durables de la vulnérabilité sociale des populations par le biais de tarifs sociaux. Dans l'esprit de la loi toutefois, la tarification sociale « $\mathrm{n}^{\prime}$ est pas conçue comme une obligation et laisse une certaine latitude aux communes qui peuvent ne pas l'instituer» (Billet, 2008).

Au demeurant, le maire garde toute liberté pour organiser la prise en charge de cette composante du service, et peut autant la concevoir dans le cadre de la politique sociale locale (comme dans le cas de la ville de Vannes) ou dans le cadre du service public de l'eau (en prévoyant par exemple un tarif différencié pour les publics vulnérables). L'une ou l'autre approche ne s'excluent pas mais la portée des mesures et des effets attendus ne relèvent pas du tout du même niveau: la tarification sociale fait partie des instruments $d$ 'internalisation de l'équité dans le management de la citadelle technique, ce que ne permet pas l'aide sociale. La prise en compte du prix de l'eau et de ses effets sur l'accès au service des publics vulnérables dans un contexte de monopole naturel fait partie des questions trop souvent ignorées ou considérées comme subsidiaires dans l'évaluation de la performance des services publics d'eau (Ernst, 1994).

En France où la gestion de l'eau n'est pas privatisée, à l'inverse des pays anglo-saxons, la responsabilité de l'acteur politique local est centrale. Dans le contexte actuel de désengagement accru de l'État central et en l'absence de régulation démocratique des services publics d'eau, c'est bien l'élu qui est le « patron» mandaté par les citoyens pour concevoir et gouverner la politique locale de l'eau.

Au-delà cependant, on peut penser que l'idée d'une responsabilité partagée à la fois par l'acteur politique, les distributeurs d'eau et les usagers citoyens, soit une condition nécessaire pour véritablement initier un modèle de management des services publics d'eau imprégné des principes du développement durable.

\section{La responsabilité de la cohésion sociale dans le secteur de l'eau est une responsabilité partagée}

À l'heure où le discours sur la "responsabilité sociale » des entreprises fait office de nouveau leitmotiv dans la sphère du privé, il serait difficile de penser que la citadelle technique dans 
le monde des services publics d'eau s'affranchisse de l'exigence de cohésion sociale. La prise en charge de la vulnérabilité des populations dans le domaine de l'accès à l'eau n'équivaut pas à « faire du social », elle fait partie de ses missions vis-à-vis de la société et renvoie aux nouveaux enjeux posés aux services publics et à leur "fonction de veille sociétale " (Decreton, 1999). La responsabilité des services publics à produire du lien social n'est pas une option: « ces activités (47) de prestation, de distribution, d'équipement, qu'elles prennent ou non la forme marchande, sont toujours marquées par une dimension fondamentale: elles contribuent dans tous les cas à une distribution ou à une redistribution des richesses et des biens, en faveur de zones géographiques, de groupes sociaux, de familles ou d'individus; et ce au nom d'intérêts collectifs, d'objectifs de solidarité, d'équité, d'assurance collective, de cohésion sociale » (Strobel, 1995). Dans un contexte de monopole local conférant véritablement aux acteurs un confort budgétaire et même plus, I'hypothèse d'un prix de l'eau loyal et légitime n'est pas avérée (Tsanga Tabi, 2003) et la question du niveau des prix de l'eau et de son caractère abordable pour les populations vulnérables ne peut pas être évacuée des solutions à mettre en œuvre. Parce qu'elle facilite la mise en évidence de ces enjeux, dans la mesure où elle appelle à une gestion des interfaces entre l'économique et le social, I'approche par le développement durable dans la gestion des services publics est utile, mais elle passe forcément (48) par le management de la citadelle technique et donc par l'implication des acteurs gestionnaires.

Le mode partenarial testé par les acteurs dans le cadre des expériences décrites est d'abord un apprentissage qui jusque-là s'est fait sur le mode minimaliste. Cet apprentissage initie cependant la construction de nouvelles représentations du service public d'eau et de ses enjeux où le social devient un objet de gestion en soi. Ce partenariat traduit aussi à l'intérieur du service les faiblesses de la catégorie galvaudée de « client », qui n'est pas pertinente pour rendre compte des spécificités du segment d'usagers que représentent les publics vulnérables, et pour lesquels les expertises et savoir-faire traditionnels sont inadaptés. Cet apprentissage marque également les limites du paradigme de l'accès marchand au service et pose la question de la frontière de la relation marchande inconditionnelle dans l'accès et la préservation de l'accès à l'eau des populations les plus pauvres. Ces remises en cause ne sont pas anodines car elles suggèrent des solutions et des voies nouvelles tournées vers ces catégories vulnérables (connaissance fine de ces usagers, tarifs sociaux et subventions croisées, nouvelles modalités de partage des gains de productivité et de la rente de monopole, accueil et relation de service dédiée...) en vue d'un management garantissant les conditions d'une inclusion au service sans qu'il soit question de mettre fin en soi au principe « marchand » du service.

Il y a, enfin, grand besoin de modes de résolution de ce problème d'exclusion au service selon l'idéal démocratique en prenant en compte tous les intérêts en présence au sein du système d'acteurs, et notamment ceux de l'usager-citoyen, l'éternel exclu des débats de fond. La question de la démocratie participative est remise à l'ordre du jour et réinterroge l'utilité et I'efficacité des modalités de fonctionnement actuelles des commissions consultatives d'usagers initiées dans le domaine de la gestion locale de l'eau depuis la loi relative à l'administration territoriale de la République (loi ATR) de 1992.

Lorsque l'organisation politique, les critères institutionnels du service public ne se suffisent pas par eux-mêmes, le service public d'eau implique nécessairement la recherche d'une finalité sociale dans un monde nanti en termes d'infrastructures et de techniques en services publics essentiels, mais où, malgré tout, les fossés se creusent et s'élargissent entre population solvable et population non solvable. Aussi, le besoin d'équité estil encore plus grand; équité dont on peut penser qu'elle est plus facile à décider tout au moins dans les pays riches et réputés démocratiques que dans les pays paurres.

Au final, il ressort que c'est la prise en compte sans détour par les acteurs de l'eau des conséquences de la vulnérabilité des populations en termes d'exclusion à ce service public essentiel, qui inaugurerait l'émergence d'un nouveau modèle de management des services publics d'eau. La finalité du management des services publics d'eau contemporains réside " aussi » dans la réalisation de l'enjeu de cohésion sociale, « tout en » conservant son statut de service public financé par le prix payé par l'usager.

Marie TSANGA TABI, Docteur en sciences de gestion (management public) et chercheur au sein de I'U.M.R Cemagref-ENGEES en

"Gestion des Services Publics». Ses travaux portent sur l'évaluation de l'action publique locale et l'analyse de la performance des organisations publiques avec pour terrain d'application les services publics d'eau. Le 2e volet de sa recherche, qui prolonge ce premier axe, concerne l'accès à l'eau des publics vulnérables dans les pays $d u$ Nord et les pays du Sud. Cemagref, U.M.R. GSP, 1, quai Koch, F-67070 Strasbourg _marie.tsanga@engees.u-strasbg.fr 


\section{NOTES}

(1) Les acteurs de l'eau désignent ici la catégorie « politique » représentée par l'autorité organisatrice du service public d'eau et la catégorie gestionnaire que sont les opérateurs de réseaux.

(2) La situation des pays pauvres comparativement à celle des pays riches est de ce point de vue particulièrement parlante.

(3) Phénomène désigné par les Anglo-Saxons par la notion $\mathrm{d}^{\prime}$ « affordability».

(4) Si la valeur de ce seuil n'est pas stabilisée, elle varie finalement peu d'une étude à une autre. Généralement calculé en prenant en compte la part des dépenses en eau sur le 1er décile inférieur du revenu des ménages, I'OCDE (2003) par exemple fait référence à une valeur moyenne de $4 \%$ établie sur un échantillon de pays de l'OCDE. Le seuil de 3\% est repris en France dans les travaux de Smets (2007), ainsi que dans les études de Sawkins and Dickie (2005) au Royaume-Uni. L'OMS (2003) retiendrait la valeur de $5 \%$.

(5) Au seuil de $60 \%$ du revenu médian, le taux de pauvreté est passé de 13,5 à $12,2 \%$ entre 1996 et 2004. En 2004, on dénombrait 6,9 millions de personnes pauvres (dans: Portrait social de la France, INSEE, dixième édition).

(6) Dont $22 \%$ de jeunes diplômés, $18 \%$ de femmes seules avec enfants, $13 \%$ de couples dont la moitié avec enfants.

(7) Chiffre donné par Smets dans un rapport de 2007.

(8) Article 93 dont le principe vise à généraliser la situation d'abonnés à l'ensemble des usagers d'eau.

(9) Charte Solidarité Eau du 6 novembre 1996.

(10) Réforme introduite par la loi n²004-809 du 13 août 2004 relative aux libertés et responsabilités locales et dont le décret d'application a été publié en mars 2005. Cette nouvelle loi traduit principalement le désengagement de l'État dans la gestion de la solidarité nationale et introduit le principe $d^{\prime}$ un fonds unique pour l'ensemble des services publics essentiels (eau, énergie et téléphone).

(11) Expression tirée de nos entretiens menés à Nantes en 2001 avec différents représentants de la hiérarchie gestionnaire du service.

(12) Cette formulation en termes de " paradigme de l'accès marchand » renvoie à la vision actuelle et très répandue de la relation marchande au service inhérente à la diffusion de la logique de marchandisation du service dont I'application inconditionnelle en fait une modalité exclusive et indifférenciée de l'accès au service public d'eau, quel que soit l'usager.

(13) Les décisions des villes de Vénissieux, Saint-Denis et la commune de Champigny-sur-Marne notamment ont été qualifiées de légales par les tribunaux administratifs. Nous n'avons pas pu obtenir d'informations sur les décisions prises pour les autres communes citées dans notre article dont les arrêtés étaient également contestés par la préfecture.

(14) Il est peut-être trop tôt pour dégager une ligne générale de doctrine de ces différents arrêts des tribunaux administratifs.

(15) Décret 2005-212 du 2 mars 2005 relatif aux fonds de solidarité pour le logement.

(16) Certaines modalités des règlements intérieurs des FSL, qui imposent de régler une partie de la dette d'eau pour pouvoir bénéficier d'une aide, peuvent aboutir, en cas de non-respect, à laisser l'usager dans sa situation d'impayé et à subir une coupure d'eau.

(17) Direction de la recherche, des études, de l'évaluation et des statistiques: "L'action sociale des communes de 5000 à moins de 20000 habitants ", Études et Résultats n³07, Ministère de la santé, avril 2004.

(18) L'analyse de l'expérience de Vannes se base sur des entretiens et données statistiques recueillis en 2003, qui n'ont pu être actualisés pour des raisons de non-disponibilité des acteurs. Lors d'un échange récent, le directeur du service nous a confirmé que le fonctionnement de ce dispositif était resté identique dans ses principes.

(19) Lors d'entretiens récents menés avec les FSL de Rouen, Lille et Saint-Étienne, les acteurs sociaux confirmaient que les modalités d'intervention des distributeurs d'eau privés avaient peu varié sur le fond.

(20) La différenciation entre abonnés d'eau renvoie en principe à la distinction habitat individuel/habitat collectif; le statut d'abonné n'étant accordé qu'aux seuls usagers résidant en habitat individuel. Pour les usagers relevant de l'habitat collectif, I'abonné est le propriétaire de l'immeuble.

(21) Cette population étudiante présentant la particularité d'être présente moins d'un an.

(22) Chiffres 2003, entretiens avec le directeur de l'eau et de l'assainissement de la régie municipale de Vannes.

(23) Chiffre d'octobre 2003, entretiens avec le directeur du service de l'eau. Quoique ce chiffre soit variable d'un mois à I'autre, il est représentatif de l'éventail du risque de non-recouvrement des factures d'eau sur la ville de Vannes.

(24) Ce qui n'est plus le cas aujourd'hui, le principe de mensualisation des factures est en place depuis 2006.

(25) Implication dont l'enjeu relève d'actions de lobbying bien connues dans l'analyse des jeux de pouvoirs s'exerçant entre acteurs au sein de la gestion de l'eau.

(26) Dossiers traités par la Lyonnaise des Eaux, la Saur et Véolia Eaux. 
(27) Issue de la loi $n^{\circ} 2004-809$ du 13 août 2004 relative aux libertés et responsabilités locales qui a organisé la fusion des FSE avec les fonds " énergie » et téléphone au sein du FSL.

(28) La formule de l'abandon de créances négociée par la FP2E lors de l'élaboration de la charte correspond de fait à un dégrèvement accordé à l'usager sur sa facture d'eau initiale. Le distributeur renonce en partie ou en totalité à la part de créance qui lui est due et la déduit du montant de la facture d'eau de I'usager. Son montant est fixé à 0,15 € par abonné au démarrage avec la charte et sera réactualisé à 0,20 € lors du passage à la version convention en avril 2000. Aujourd'hui, ce montant est de 0,30 €/abonné.

(29) Selon I'INSEE, un ménage moyen (quatre personnes) consomme $120 \mathrm{~m}^{3}$, soit une dépense moyenne annuelle de 228,67 et 304,90 € sur cette période.

(30) D’après Maillard, 2000, p. 24.

(31) Le montant du plafond de ressources mensuelles en vigueur début 2001 était le suivant: 381,12 € pour une personne seule; 533,57 € pour deux personnes (couples, parents et 1 enfant) ; + 106,71 € par personne à charge supplémentaire.

(32) La part "État » qui représente au moment de l'étude en moyenne $20 \%$ de la facture d'eau, correspond aux diverses taxes (TVA) et redevances reversées aux organismes publics (Agences de bassin, Voies navigables de France).

(33) Fonction qu'il assumait au moment de l'interview en 2001.

(34) La conception égalitaire de l'accès au service public d'eau est fondée sur une différence de situation objective entre usagers à l'égard de la consommation du produit, qui seule est susceptible de justifier une différence de traitement notamment en termes de prix: des tarifs différents peuvent être appliqués aux usagers domestiques, aux industries et commerces et aux établissements publics. La jurisprudence relative à l'eau est plus d'une fois venue confirmer ce principe de discrimination entre usagers (arrêts Vidal, 1981; Paraiso, 1983; Bachelet, 1991) — Mbacké Cissé, 1996, Le service public, ses principes fondamentaux et l'activité de distribution d'eau potable. Éléments d'une évaluation de la performance de gestion, mémoire de D.E.S.S d'administration des collectivités locales, Laboratoire Gestion des Services Publics.

(35) Cité par Affichard, 1995, p. 289.

(36) Palier, 1998, p. 33.

(37) Interview de l'acteur social représentant la DDASS du Gers (2002).

(38) Les dispositifs locaux de solidarité-Eau, tel celui de la ville de Vannes, relèvent de l'aide sociale facultative et non de I'aide sociale obligatoire ( $c f$. site du CCAS de la ville de Vannes). Par ailleurs, si les dispositifs départementaux FSL engagent le Conseil Général, ils ne sont pas coercitifs pour les services publics d'eau.

(39) Le XXe siècle, qui a vu I'achèvement de l'objectif de généralisation de la desserte, a rendu obsolète le maintien d'un mécanisme destiné à développer l'accès dans les zones non couvertes.

(40) Ces actions d'aide d'urgence ou de solidarité dans les domaines de l'eau et de l'assainissement peuvent être financées par le prix de l'eau dans la limite de 1\% du budget des services.

(41) Rapport de la mission Denoix de Saint Marc, p. 14.

(42) Ibid. p. 49.

(43) Rapport dont l'intérêt réside dans le fait qu'il porte en particulier sur les services publics en réseau.

(44) «En tant que structure de sens, le référentiel articule quatre niveaux de perception du monde qui doivent être distingués mais dont les liens sont évidents: des valeurs, des normes, des algorithmes et des images » Muller, 2006, p. 372.

(45) Qui postule notamment que « chaque personne physique, pour son alimentation et son hygiène, a le droit d'accéder à l'eau potable dans des conditions économiquement acceptables par tous »

(46) À partir de 2010 seulement.

(47) « ces » renvoie aux services publics.

(48) Si l'on veut dépasser le stade du principe et de la rhétorique.

\section{BiBLIOGRAPHIE}

AfFiCHARD J., 1995, "Management public et traitement des usagers ", in Le service public?, La voie moderne, Colloque de Cerisy, pp. 281-294.

AGGERI F., 2001, «Développement durable et gouvernement de I'environnement: la formation d'un nouvel espace d'action collective ", communication au Séminaire Condor, 13 décembre.

Billet P., 2008, « La tarification « sociale » de l'eau. Perspectives offertes par la LEMA du 30 décembre 2006 », séminaire scientifique et technique de I'ENGEES du 22 janvier 2008.

BIPE/FP2E, 2006, "Les services collectifs d'eau et d'assainissement en France », Données économiques, sociales et techniques, 2e édition

Boiteux M., 1996, Concurrence, régulation, services publics Variations autour du cas de l'électricité, Futuribles.

BULLETIN JURIDIQUE DES COLLECTIVITÉS LOCAlES, 2005, un maire 
peut-il demander l'interruption de la desserte électrique d'une construction irrégulière déjà raccordée au réseau? $\mathrm{n}^{\circ} 3$, pp 170-172.

BRONOS S., 2007, « Access to water services for the urban poor in Europe: characterising and considering vulnerable groups in French and English publics policies ", Thesis of Master of Science of Cranfield University, Joint research unit Cemagref-Engees in Public Services Management.

CASTEL R., 1995, Les métamorphoses de la question sociale, Fayard, Paris

Denolx de Saint Marc R., 1996, Le service public, Rapport au Premier ministre, La Documentation française, Collection des rapports officiels.

ERNST J., 1994, Whose utility? The social impact of public utility privatization and regulation in Britain, Public Policy and Management, Open university Press.

DeCreton S. (sous la dir.), 1999, Service public et lien social, L'Harmattan, Logiques juridiques

DuBOIS J-L., MAHIEU F-R, 2002, « La dimension sociale du développement durable: réduction de la pauvreté ou durabilité sociale? ", in Développement durable? Doctrines, Pratiques, Évaluations, Textes réunis et présentés par J-Y. Martin, IRD, pp. 73-94.

FitCH, M., PriCE H., 2002, Water poverty in England and Wales. Chartered Institute of Environmental Health

GADREY J, 1998, Les prestations sociales de La Poste, rapport coordonné par La Poste

Strobel P., D. Kaisergruber, 1996, "Service public, fin de siècle. Contraintes européennes et défi de la pauvreté ", in Le service public en recherche, quelle modernisation, Catherine Grémion et Robert Fraisse (dir.), La documentation française, pp. 21-38.

Laumonier C, Giffo-Levasseur A-M, Rousselot L., 2006, Étude sociologique dans le cadre de l'individualisation des abonnements au service d'eau potable, CSTB-Gers, rapport à la Direction de l'eau de Nantes-Métropole.

LeNOIR R., 1974, Les Exclus, un Français sur dix, Paris, Le Seuil.

MAILLARD J. DE, 2000, « Le partenariat en représentations: contribution à I'analyse des nouvelles politiques sociales territorialisées » in Revue politiques et Management Public, Vol 18, Septembre 2000, n³, pp 21-41.

Muller P., 2006, "Le référentiel », Dictionnaire des politiques publiques, sous la direction de L. Boussaguet, Jacquot S., Ravin N., 2e édition, pp. 370-376

OCDE, 2003, «Problèmes sociaux liés à la distribution et à la tarification de l'eau », Éditions OCDE.

PAlier B., 1998, « La référence au territoire dans les nouvelles politiques sociales », Revue politiques et Management Public, volume vol 16, n³, pp 13-41.

Paugam S., 1991, La Disqualification sociale. Essai sur la nouvelle pauvreté, Paris, PUF.

Périvier H., 2006, "Quel sort pour les allocataires des minimas sociaux?» La lettre de l'OFCE, $\mathrm{n}^{\circ} 273$.

SAWkINS J., DICKIE V.A., 2005, "Affordability of Household Water and Sewerage Services in Great Britain », Fiscal Studies, vol. 26, n², pp. 225-244 (Accessed: 05/06/2007) http://www.blackwell-synergy.com/doi/pdf/10.1111/j.14755890.2005.00011.x

SMETS H., 2007, La prise en charge des dettes d'eau des usagers démunis en France, Académie de l'Eau.

Strobel P., 1995, "Services publics et cohésion sociale ", Recherches et Prévisions n42, pp. 7-16.

ReYNAUD A., 2007, Social policies and private sector participation in water supply - the case of France, report for the United Nations Research Institute for Social development.

TSANGA TABI M., 2003, "Théorie et réalité du service public local: le cas de la distribution d'eau potable ", thèse de doctorat en sciences de gestion, Université de Paris X, $771 \mathrm{p}$.

TSANGA TABı M., 2004, Quelle prise en compte du développement durable dans l'évaluation de la performance des services publics d'eau potable? Premiers éléments de réflexion à partir de l'analyse de trois cas de collectivités, Rapport au Ministère de I'Environnement et du Développement Durable.

TSANGA TABI M., 2004, "La place du développement durable dans le nouveau référentiel des services publics d'eau potable, Débats autour de l'évaluation des services publics marchands », in La dynamique de l'évaluation face au développement durable, (sous la dir de C. Offredi), L'Harmattan, pp. 151-165.

TSANGA TABI M., 2006, «Entre client-centrisme et droit à I'eau: le dilemme posé par l'usager non solvable dans la gestion des services publics marchands », Revue Politiques et Management Public, vol 24, n4, pp. 69-87.

Tsanga TABı M., Le Palabe E., 2007, « La politique publique de maintien à l'eau des publics défavorisés en France: état des lieux de la mise en place des dispositifs sur le territoire national » le 4 pages, édition Cemagref. 